Running Head: IMPLICIT COGNITIVE PROCESSES

\title{
IMPLICIT COGNITIVE PROCESSES IN BINGE-EATING DISODER AND OBESITY
}

\author{
Brauhardt, Anne, MSc, Rudolph, Almut, PhD, and Hilbert, Anja, PhD \\ Leipzig University Medical Center, Integrated Research and Treatment Center \\ AdiposityDiseases, Medical Psychology and Medical Sociology
}

Location where work was conducted: Philipps University of Marburg, Marburg, Germany

Correspondence concerning this manuscript should be addressed to Anne Brauhardt, MSc, Leipzig University Medical Center, Integrated Research and Treatment Center AdiposityDiseases, Medical Psychology and Medical Sociology, Philipp-Rosenthal-Strasse 27, 04103 Leipzig (Germany). Email: anne.brauhardt@medizin.uni-leipzig.de, Phone: +49 34197 15364, Fax: +49 3419715378.

The final publication is available at http://dx.doi.org/10.1016/j.jbtep.2014.01.001. (C) [2014]. This manuscript version is made available under the CC-BY-NC-ND 4.0 license http://creativecommons.org/licenses/by-nc-nd/4.0/ 
IMPLICIT COGNITIVE PROCESSES

\begin{abstract}
Objectives: Binge-eating disorder (BED) is characterized by recurrent binge eating episodes, associated eating disorder and general psychopathology, and commonly occurs in obese individuals. Explicit self-esteem and explicit weight bias have been linked to BED, while little is known about implicit cognitive processes such as implicit self-esteem and implicit weight bias.
\end{abstract}

Methods: Obese participants with BED and an individually matched obese only group $(\mathrm{OB})$ and normal weight control group $(\mathrm{CG}$; each $\mathrm{N}=26)$ were recruited from the community to examine group differences and associations in explicit and implicit selfesteem and weight bias, as well as the impact of implicit cognitive processes on global eating disorder psychopathology. Implicit cognitive processes were assessed using the Implicit Association Test.

Results: Significantly lower explicit self-esteem, as well as higher exposure to explicit weight bias, compared to CG and OB was found in the BED group. All groups showed positive implicit self-esteem, however, it was significantly lower in BED when compared to CG. BED and CG demonstrated equally high implicit weight bias whereas OB did not. Explicit and implicit measures were not significantly correlated. Global eating disorder psychopathology was predicted by explicit and implicit self-esteem.

Conclusions: The results of the present study add to the importance of implicit selfesteem and implicit weight bias beyond explicit measures in BED, while both were previously shown to be associated with onset and maintenance of BED. In conclusion, implicit cognitive processes should be focused on in interventions for BED to investigate their impact on psychological treatments.

Key words: implicit self-esteem, explicit self-esteem, implicit weight bias, explicit weight bias, binge-eating disorder, obesity 
IMPLICIT COGNITIVE PROCESSES

\section{INTRODUCTION}

Since the publication of research criteria for binge-eating disorder (BED) in the Diagnostic and Statistical Manual of Mental Disorders (DSM-IV; American Psychiatric Association [APA], 1994), substantial research on various aspects of BED led to the inclusion of BED in the recently published DSM-5 (APA, 2013). BED is characterized by recurrent binge eating episodes including eating large amounts of food accompanied by a sense of lack of control over eating that occurs in the absence of compensatory behaviors. BED is associated with lower quality of life, and increased eating disorder and general psychopathology. Further, BED commonly occurs in obese individuals, however, BED and obesity (defined as body mass index $[\mathrm{BMI}] \geq 30 \mathrm{~kg} / \mathrm{m}^{2}$ ) represent distinct phenomena (APA, 2013).

Various cognitive processes have been conceptualized as factors for the onset and maintenance of BED (e.g., Fairburn, Cooper, \& Shafran, 2003; Wilfley, Pike, \& StriegelMoore, 1997). Further, substantial evidence highlighted the importance of self-esteem (SE) and exposure to weight bias (WB) as cognitive processes contributing to BED. Low SE has repeatedly been shown to be a predictor for BED in cross-sectional and longitudinal studies (Goldschmidt, Wall, Loth, Le Grange, \& Neumark-Sztainer, 2012; Grilo, White, \& Masheb, 2012), and has also been found in obesity (Griffiths, Parsons, \& Hill, 2010). High rates of exposure to WB, defined as being stigmatized because of one's weight, repeatedly predicted binge eating (Ashmore, Friedman, Reichmann, \& Musante, 2008) and binge eating onset (Goldschmidt et al., 2012).

However, individuals might lack self-insight, deceive others or even themselves in self-report measures on SE and WB (Wilson, Lindsey, \& Schooler, 2000). Therefore, previous research provided an elaborated theoretical dual-model approach comprising two structurally distinct systems of information processing that encompasses explicit and implicit 
evaluations (e.g., Strack \& Deutsch, 2004). Thus, explicit SE and explicit WB are understood as deliberate, accessible and mentally represented information in a reflective system that can be assessed with self-report measures. However, implicit SE and implicit WB are automatic and not necessarily conscious evaluations in an impulsive system. A reliable and valid measure of implicit evaluations is the Implicit Association Test (IAT; Greenwald, McGhee, \& Schwartz, 1998). Previous research revealed typically weak correlations between implicit and explicit measures (Hofmann, Gawronski, Gschwendner, Le, \& Schmitt, 2005). Furthermore, implicit measures have been found to demonstrate additional predictive power as they predicted spontaneous impulsive behavioral responses that explicit measures were not able to predict (e.g., Roefs et al., 2011; Rudolph, SchröderAbé, Riketta, \& Schütz, 2010). Thus, it is necessary to examine explicit and implicit cognitions in BED.

Research on implicit SE in eating disorders is rare and findings have been inconsistent. While implicit SE did not differ in restrained and unrestrained eaters (Hoffmeister, Teige-Mocigemba, Blechert, Klauer, \& Tuschen-Caffier, 2010), participants with bulimia nervosa were found to have higher implicit SE compared to healthy controls (Cockerham, Stopa, Bell, \& Gregg, 2009). In contrast, lower - albeit positive - implicit SE when compared to healthy controls was reported in patients with depression (Risch et al., 2010) and body dysmorphic disorder (Buhlmann, Teachman, Naumann, Fehlinger, \& Rief, 2009). Previously, implicit WB was demonstrated in normal weight (Watts \& Cranney, 2009) and obese samples (Carels et al., 2010). In BED, implicit WB has not been studied yet, however, implicit WB was found to be a predictor of binge eating in weight loss treatment seeking individuals (Carels et al., 2010).

Based on the findings above, our aim was to investigate effects of eating disorder psychopathology and obesity on explicit and implicit cognitive processes. Thus, we 
examined obese participants diagnosed with BED (BED group), obese participants without an eating disorder diagnosis (OB group), and normal weight participants without an eating disorder diagnosis in a control group (CG). First, we expected the BED group to show lower explicit SE and to explicitly report higher exposure to WB compared to the OB group and CG. Additionally, when comparing the BED group to the OB group and the CG we predicted lower implicit SE but no differences in implicit WB. Second, we expected weak correlations between explicit and implicit measures of SE and WB. Finally, we explored whether and to what extent implicit cognitive processes possessed incremental validity over and above explicit cognitive processes in the prediction of global eating disorder psychopathology.

\section{METHOD}

\subsection{Participants and Recruitment}

Seventy-eight participants (63 women, $80.8 \%$ ) older than 18 years of age and with sufficient German language skills were recruited from the community and offered $€ 40$ for participation. Participants in the three groups were individually matched according to sex, age, and education, revealing three groups of equal sizes $(N=26)$. Additionally, the BED and OB group participants were matched according to their BMI.

Inclusion criteria in the BED group were a diagnosis of BED according to DSM-5 criteria (APA, 2013), and a BMI in the obese category (BMI $\geq 30 \mathrm{~kg} / \mathrm{m}^{2}$ ). Inclusion in the OB group required a BMI $\geq 30 \mathrm{~kg} / \mathrm{m}^{2}$, and the $\mathrm{CG}$ had to be of normal weight $\left(18.5 \mathrm{~kg} / \mathrm{m}^{2}<\right.$ BMI $<24.9 \mathrm{~kg} / \mathrm{m}^{2}$ ). Exclusion criteria in the OB group and CG included reports of any episodes of binge eating or inappropriate compensatory behavior within the last six months. 


\section{$2.2 \quad$ Measures}

Eating Disorder Examination (EDE). The diagnostic items of the semi-structured EDE interview (German version: Hilbert, Tuschen-Caffier, \& Ohms, 2004) were used for BED diagnosis. Good reliability $(.73 \leq$ Cronbach's $\alpha \leq .93)$ and validity have been established for the original and German version.

Eating Disorder Examination-Questionnaire (EDE-Q; German version: Hilbert, Tuschen-Caffier, Karwautz, Niederhofer, \& Munsch, 2007). Specific eating disorder psychopathology within the past 28 days was assessed with the 28 item EDE-Q $(0=$ not at all to $6=$ markedly) comprised of four mean scores for subscales (restraint, eating concern, weight concern, and shape concern) and global eating disorder psychopathology. The EDEQ demonstrated excellent reliability (Cronbach's $\alpha=.95$ ) in the current study.

Rosenberg Self-Esteem Scale (RSES; German version: von Collani \& Herzberg, 2003). Explicit self-esteem, the overall liking of oneself, was measured using the sum score of the ten item RSES ( $1=$ strongly disagree to $4=$ strongly agree $)$. The RSES showed excellent reliability (Cronbach's $\alpha=.92)$.

Stigmatizing Situations Inventory (SSI; Myers \& Rosen, 1999). The SSI was used to assess exposure to WB. For a wide range of social situations participants indicated the frequency with which they experienced stigmatization $(0=$ never to $9=$ daily $)$ because of their weight over their life span (e.g., negative comments from children, being stared at, being avoided, being excluded, being ignored because of one's weight). A global mean score over all 50 items was computed. Excellent reliability (Cronbach's $\alpha=.95$ ) was obtained for the German version that was based on a translation and back-translation procedure (Hilbert, unpublished manuscript).

Brief Symptom Inventory (BSI; German version: Franke, 2000). T-standardized mean scores of the Global Severity Index (GSI) calculated from the 53 items of the BSI $(0=$ not at 
all to 4 = extremely) indicated general psychopathological impairments during the last week on nine dimensions (somatisation, obsession-compulsion, interpersonal sensitivity, depression, anxiety, hostility, phobic anxiety, paranoid ideation, psychoticism). The BSI demonstrated excellent reliability (Cronbach's $\alpha=.96$ ).

Implicit Association Test (IAT). As a measure of implicit SE and implicit WB, the IAT (Greenwald, et al., 1998) was administered using Inquisit Millisecond Software (Draine, 2004). The IAT as a computerized measure assessed the relative strength of associations between one pair of opposing attribute categories and one pair of opposing target categories. The IAT established good reliability $(.80<$ Cronbach's $\alpha<.85)$, convergent, discriminant, and predictive validity (Rudolph, Schröder-Abé, Schütz, Gregg, \& Sedikides, 2008; Rudolph, Schröder-Abé, Riketta, \& Schütz, 2010).

In the Self-Esteem IAT (SE-IAT) participants were instructed to categorize attribute stimuli as positive or negative and target stimuli into a self or other category (Greenwald \& Farnham, 2000; stimuli developed by AH). Attribute stimuli contained four positive (e.g., loved, smart) and negative adjectives (e.g., awful, stupid). Target stimuli included four selfrelated (e.g., me, myself) and other-related stimuli (e.g., they, their). An idiographic variant of the SE-IAT was used with the participant's first name presented as one self-related stimulus. Similarly, in the Weight Bias IAT (WB-IAT) participants were asked to categorize attribute and target stimuli. The only difference was that target categories were thin (e.g., underweight, slim) and fat (e.g., plump, overweight; Carels et al., 2010). In the two critical blocks of the IAT, participants were asked to press one of two keys to classify the four types of stimuli into their respective categories. In one block, the categories were paired in a compatible way, and in the other block, in an incompatible way. Compatible block pairings were self-positive | other-negative (SE-IAT) and thin-positive |fat-negative (WBIAT), incompatible block pairings were other - positive | self-negative (SE-IAT) and thin- 
negative $\mid$ fat-positive (WB-IAT). For SE-IAT and WB-IAT, faster responding in the compatible block relative to the incompatible block is taken as evidence of automatic preference for self - positive over self - negative and fat - negative over fat - positive, respectively. Thus, for both IATs separately, the IAT index was calculated as the difference between the two combined blocks using the new scoring algorithm (D-score; Greenwald, Nosek, \& Banaji, 2003).

\section{$\underline{2.3 \quad \text { Procedures }}$}

Approval for the study was granted by the German Psychological Society's ethics committee. Participants were screened via telephone interview with EDE items (Hilbert et al., 2007) and sociodemographic questions to determine eligibility regarding inclusion and exclusion criteria. Based on this information, individuals for the OB group and CG were selected to individually match the BED participants' characteristics with minor deviations being considered acceptable. Following the screening procedure, self-report measures were sent to the participants. During the laboratory assessment, every participant was seen individually for 1.5 hours by a trained research assistant. After obtaining informed consent, the diagnostic part of the session included the assessment of the BMI and the EDE interview to determine the presence of a BED diagnosis for the BED group or absence of any eating disorder diagnosis for the OB group and CG. Subsequently, in the experimental part of the session, the IATs were conducted.

\subsection{Data Analytic Plan}

Hypothesized group differences in explicit and implicit measures between matched groups were analyzed using univariate repeated measures ANOVAs with subsequent posthoc Bonferroni tests. Preferences for self - positive over self - negative and fat - negative 
over fat - positive were further analyzed with one-sample $t$-tests to compare D-score means with the theoretical mid-point of the scale. Pearson correlations were used to determine relations between measures. Hierarchical multiple regressions were run to assess impact of group membership (dummy coded with CG as reference group; block 1), explicit and implicit SE (RSES, SE-IAT; block 2), and explicit and implicit WB (SSI, WB-IAT; block 3) on global eating disorder psychopathology (EDE-Q global score). Overall, significance levels were set at a two-tailed $\alpha<.05$ for all tests. Effect sizes for all analyses were calculated and interpreted as small $\left(.01 \leq \eta^{2}<.06 ; d>0.2 ; .1 \leq \mathrm{r}<.3 ; .02 \leq \mathrm{R}^{2}<.13\right)$, or medium $\left(.06 \leq \eta^{2}<.14 ; d>0.5 ; .3 \leq \mathrm{r}<.5 ; 1.13 \leq \mathrm{R}^{2}<.26\right)$, or large $\left(\eta^{2} \geq .14 ; d>0.8 ; \mathrm{r} \geq\right.$ $.5 ; \mathrm{R}^{2} \geq .26$; Cohen, 1988). Statistical analyses were performed using IBM SPSS Statistics 20.0.

\section{RESULTS}

\subsection{Sample Characteristics and Preliminary Analyses}

First, all groups were compared to test the matching procedure. No differences were found in sociodemographic variables. As expected, groups differed in BMI, eating disorder psychopathology (EDE-Q), and depression (BDI). In post-hoc Bonferroni tests, the BED group showed significantly higher mean scores in the EDE-Q subscales and global score and significantly higher sum scores in the BSI followed by the OB group and the CG (see Table 1). Participants of the BED group reported on average $13.00(S D=11.50)$ binge eating episodes in the last 28 days.

\subsection{Group Differences in Explicit and Implicit Measures}

As expected, substantial differences between the three groups were found for all selfreport measures (see Table 1). Post-hoc tests revealed lower explicit SE (RSES) in the BED 
group compared to both the $\mathrm{OB}$ group and $\mathrm{CG}$, whereas the latter two did not differ significantly. Significantly higher means for exposure to WB over the life span (SSI) were reported in the BED group, while the OB group reported higher means than the CG.

For implicit SE (SE-IAT), significant group differences were found with lower implicit SE in the BED group compared to the $\mathrm{CG}$, but not between the BED and the OB groups (see Table 1). SE-IAT D-scores in the OB and the CG groups did not differ. Furthermore, a pronounced self-positivity bias was found in all groups, revealing a general preference for self - positive over self - negative with overall large effects in the BED group $[T(25)=3.77, p=0.001, d=0.74]$, in the OB group $[T(25)=9.88, p<0.001, d=1.94]$, and in the $\mathrm{CG}[T(25)=10.00, p<0.001, d=1.96]$.

For implicit WB (WB-IAT), the overall effect was significant. In post-hoc tests, significant differences were found between the OB group and the CG with lower WB-IAT D-scores in the OB group. Interestingly, individuals in the OB group did not yield a preference of fat - negative over fat - positive $[T(25)=0.20, p=0.84, d=0.04]$, however, this preference yielded a medium effect in the BED group $[T(25)=2.13, p=0.04, d=0.42]$ and a large effect in the CG $[T(25)=4.77, p<0.001, d=0.94]$.

\section{$\underline{3.3 \quad \text { Intercorrelations of Explicit and Implicit Measures }}$}

As previously shown, implicit and explicit self-esteem were not correlated over all groups $\left(r_{S E-I A T \_R S E S}=0.13, p=0.26\right)$. Similarly, lifetime discriminating experiences and implicit WB did not correlate $\left(r_{S S I}\right.$ WB-IAT $\left.=-0.08, p=0.48\right)$. The two implicit measures were uncorrelated $\left(r_{S E-I A T \_W B-I A T}=0.07, p=0.52\right)$. 


\subsection{Prediction of Global Eating Disorder Psychopathology and Additional Analyses}

In order to determine whether and to what extent implicit cognitive processes predicted global eating disorder psychopathology, explicit and implicit measures of SE and WB were regressed on the EDE-Q global score. Across all groups (see Table 2), group membership explained a significant and large amount of variance $\left(R^{2}=0.45, p<0.001\right)$, While explicit SE (RSES), and implicit SE (SE-IAT) explained another significant amount of variance $\left(\Delta R^{2}=0.11 ; p<0.001\right)$, neither explicit WB nor implicit WB changed the overall variance explained $\left(\Delta R^{2}<0.01 ; p=0.80\right)$. More detailed, higher levels of global eating disorder psychopathology were predicted by lower explicit SE, and higher implicit SE. When entering explicit and implicit SE (Step 1), and explicit and implicit WB (Step 2) in a regression analysis for the BED group alone $(N=26)$, the first block of variables explained a significant and large amount $\left(R^{2}=0.28, p=.02\right)$ of the total variance $\left(R^{2}=\right.$ 0.34), however, only implicit SE (SE-IAT) emerged as a significant predictor of the global eating disorder psychopathology $(B=1.10, S E=0.47, \beta=0.42, p=0.03)$. Though, this finding should to be viewed as preliminary as the regression analysis was based on a small sample.

\section{DISCUSSION}

First, the present study sought to examine implicit cognitive processes in BED and obesity compared to normal weight control participants. We found lowest levels of implicit SE in obese participants with BED, and lowest levels of implicit WB in obese participants. Second, associations between implicit and explicit measures were investigated, and as hypothesized, implicit and explicit measures were only weakly correlated indicating the distinctiveness of the impulsive and reflective system. Third, we examined the incremental 
validity of implicit cognitions, and found that implicit SE predicted global eating disorder psychopathology over and above explicit cognitions.

In line with our hypothesis, the results showed significantly lower implicit SE in participants with BED when compared to normal weight controls. This finding replicates evidence for decreased - albeit positive - implicit SE in mental disorders (Buhlmann et al., 2009; Risch et al., 2010). Confirming previous research (Watts \& Cranney, 2009), our investigations on implicit WB revealed high expressions of negative attitudes towards obesity in the normal weight group. Surprisingly, within obese participants no implicit WB was identified which might be attributed to a higher mean BMI in the OB group when compared to previous studies. In accordance with previous findings, lower levels of implicit WB in participants with higher BMI was evident (Watts \& Cranney, 2009). For the obese participants with BED, however, we found the expected expressions of negative attitudes towards obesity which might be attributed to higher levels of eating disorder and general psychopathology as well as higher exposure to explicit WB in obese individuals with BED compared to obese individuals without BED (Wonderlich, Gordon, Mitchell, Crosby, \& Engel, 2009). Corroborating existing literature, we found implicit and explicit measures of SE and WB not to be associated underlining the distinctiveness of the reflective and impulsive system (Hofmann, et al., 2005; Rudolph et al., 2008).

The major strength of this study is the well controlled sample that ruled out effects of sex, age, education, and BMI on the dependent variables. Additionally, with its thorough psychopathological assessment the present study significantly differs from previous research as BED was assessed via clinical expert interview to confirm the diagnosis. Furthermore, this study was the first that assessed explicit and implicit WB in BED using reliable and valid measures. 
Our results also need to be interpreted with regard to several limitations. First, one might argue that the diagnostic interview assessment at the beginning of the laboratory assessment might have increased the accessibility of weight-related cognitions, and thus have affected subsequent IAT performance. However, accumulated evidence suggests that task order effects are rather minimal (Hofmann et al., 2005). We therefore kept task order constant to minimize method variance between participants. Second, previously published clinical norms (e.g., Aardom, Dingemans, Slof Op’t Landt, \& Van Furth, 2012) reported lower levels of global eating disorder psychopathology for obese individuals and individuals with eating disorders than we found for our BED and OB group. However, the level of eating disorder psychopathology in the OB group was significantly higher compared to community-based samples (Hilbert et al., 2012). The apparently lower levels in the BED and the OB group could be attributed to the non-treatment seeking sample in the present study while clinical norms typically assessed individuals currently receiving treatment. Third, our exclusion criteria for the OB group and the CG mainly targeted current BED diagnosis while lifetime eating disorder diagnoses and/or comorbid psychopathology were not assessed. Nevertheless, both groups did not report binge eating episodes or inappropriate compensatory behaviors within the last six months or current general psychopathological impairments. Likewise, we did not screen for previous or current mental disorders in our BED group as high comorbidity rates of BED and affective disorders exist (Hilbert et al., 2011).

The application of dual-model approaches in BED might need further attention, especially as our results reveal a rather puzzling pattern. The initial evidence points to the potential incorporation of the cognitive model of eating disorders and recent dual-model approaches of information processing As implicit measures often reflect dysfunctional beliefs (e.g., the self being associated with negative attributes) specified in the cognitive 
model within the impulsive system, implicit measures can reveal aspects of the dysfunctional beliefs that explicit measures cannot reveal in the reflective system. Substantial evidence demonstrated the predictive validity of implicit measures over and above explicit measures (Greenwald, Poehlman, \& Banaji, 2009; Rudolph et al., 2010), while initial evidence highlighted the clinical validity of implicit measures (Creemers et al., 2012; Vater, SchröderAbé, Schutz, Lammers, \& Roepke, 2010). Beyond our findings described above, implicit SE emerged as a significant predictor of global eating disorder psychopathology in addition to explicit SE. In detail, impaired psychopathology was associated with higher implicit SE and lower explicit SE. This rather surprising finding, however, might incorporate the fact that another empirical pattern that was repeatedly found in recent research on implicit SE: Discrepancies between explicit and implicit SE may occur, with both fragile SE (i.e., high explicit and low implicit SE) and damaged SE (i.e., low explicit and high implicit SE) being associated with psychological dysfunction and symptom severity (Schröder-Abé, Rudolph, \& Schütz, 2007; Vater, Schröder-Abé, Schütz, Lammers, \& Roepke). So far, empirical evidence of discrepant SE in participants with eating disorders is lacking, however, this study provided initial evidence that high implicit SE might not necessarily be advantageous as it could result in increased psychopathology.

Further research avenues can be derived from our results and their clinical implications. First, research on implicit cognitions would need to be extended from crosssectional designs to longitudinal studies to directly investigate their impact on the development, maintenance, and remission of BED (Wiers, Teachman, \& De Houwer, 2007). In doing so, more research on the reliability of implicit measures (i.e., sensitivity to change) is warranted. Second, further investigations on the impact of implicit cognitions on BED might help to understand psychotherapeutic processes and to identify predictors of treatment outcome and maintenance (Watts \& Cranney, 2009). Third, initial evidence also highlights 
the potential of implicit training tasks to reduce symptom severity and maladaptive behaviors in various mental conditions (Baldwin et al., 2002; Svaldi et al., 2012; Clerkin \& Teachman, 2010), however, applications in individuals with eating disorders are outstanding. An intervention task based on a classical conditioning paradigm might increase implicit selfesteem by repeated pairings of self-relevant and positive stimuli (Vartanian, Polivy, \& Herman, 2004). Developing interventions to improve implicit SE and reduce implicit WB could be considered as a novel approach within evidence-based psychological treatments for BED. So far, interventions were proposed for explicit SE within the transdiagnostic treatment of eating disorders (Fairburn et al., 2003). Interventions to cope with stigma or to reduce self-stigma were presented in treatment manuals for BED (e.g., Hilbert \& TuschenCaffier, 2010) and aimed at breaking the vicious cycle of exposure to WB, loss of motivation, and the vulnerability of binge eating. However, previous studies mainly focused on explicit variables and revealed mixed results within psychological interventions. Hence, interventions regarding implicit cognitions could further improve treatment outcomes for BED.

\section{CONCLUSION}

Significant group differences were found for implicit cognitive processes, and thus added more detailed information to previous results on explicit measures associated with onset and maintenance of BED. After demonstrating the importance of implicit cognitive processes for $\mathrm{BED}$, future investigations should incorporate dual-model approaches into cognitive models of eating disorders, further evaluate implicit measures, and investigate interventions aiming at the improvement of implicit SE and the reduction of implicit WB. In doing so, advanced state-of-the-art treatments for BED could be established. 
IMPLICIT COGNITIVE PROCESSES

\section{REFERENCES}

Aiken, L. S., \& West, S. G. (1991). Multiple regression: Testing and interpreting interactions. Thousand Oaks, CA: Sage Publications.

Aardom, J. J., Dinegmans, A. E., Slof Op't Landt, M. C. T., \& Van Furth, E. (2012). Norms and discriminative validity of the Eating Disorder Examination-Questionnaire (EDEQ). Eating Behaviors, 13, 305-309.

Ashmore, J. A., Friedman, K. E., Reichmann, S. K., \& Musante, G. J. (2008). Weight-based stigmatization, psychological distress, $\&$ binge eating behavior among obese treatment-seeking adults. Eating Behaviors, 9, 203-209.

American Psychiatric Association [APA] (1994). Diagnostic and Statistical Manual of Mental Disorders - DSM-IV (4th edition). Washington: Author.

American Psychiatric Association [APA] (2013). Diagnostic and Statistical Manual of Mental Disorders - DSM-5 (5th edition). Washington, DC: Author.

Buhlmann, U., Teachman, B. A., Naumann, E., Fehlinger, T., \& Rief, W. (2009). The meaning of beauty: Implicit and explicit self-esteem and attractiveness beliefs in body dysmorphic disorder. Journal of Anxiety Disorders, 23, 694-702.

Carels, R. A., Wott, C. B., Young, K. M., Gumble, A., Koball, A., \& Oehlhof, M. W. (2010). Implicit, explicit, and internalized weight bias and psychosocial maladjustment among treatment-seeking adults. Eating Behaviors, 11, 180-185.

Clerkin, E. M., \& Teachman, B. A. (2010). Training implicit social anxiety associations: An experimental intervention. Journal of Anxiety Disorders, 24, 300-308.

Cockerham, E., Stopa, L., Bell, L., \& Gregg, A. (2009). Implicit self-esteem in bulimia nervosa. Journal of Behavior Therapy and Experimental Psychiatry, 40, 265-273.

Cohen, J. (1988). Statistical power analysis for the behavioral (2. ed.). Hillsdale, NJ: Erlbaum. 
Creemers, D. H., Scholte, R. H., Engels, R. C., Prinstein, M. J., \& Wiers, R. W. (2012). Implicit and explicit self-esteem as concurrent predictors of suicidal ideation, depressive symptoms, and loneliness. Journal of Behavior Therapy and Experimental Psychiatry, 43, 638-646.

De Houwer, J., Teige-Mocigemba, S., Spruyt, A., \& Moors, A. (2009). Implicit measures: A normative analysis and review. Psychological Bulletin, 135, 347-368.

Draine, S. (2004). Inquisit 2.05.0401 [Computer Software]. Seattle: Millisecond Software.

Fairburn, C. G., Cooper, Z., \& Shafran, R. (2003). Cognitive behaviour therapy for eating disorders: A 'transdiagnostic' theory and treatment. Behaviour Research and Therapy, $41,509-528$.

Franke, G. H. (2000). Brief Symptom Inventory (BSI). Goettingen, Germany: Beltz.

Goldschmidt, A. B., Wall, M., Loth, K. A., Le Grange, D., \& Neumark-Sztainer, D. (2012). Which dieters are at risk for the onset of binge eating? A prospective study of adolescents and young adults. Journal of Adolescent Health, 51, 86-92.

Greenwald, A. G., \& Farnham, S. D. (2000). Using the implicit association test to measure self-esteem and self-concept. Journal of Personality and Social Psychology, 79, 1022-1038.

Greenwald, A. G., McGhee, D. E., \& Schwartz, J. L. (1998). Measuring individual differences in implicit cognition: The implicit association test. Journal of Personality and Social Psychology, 74, 1464-1480.

Greenwald, A. G., Nosek, B. A., \& Banaji, M. R. (2003). Understanding and using the Implicit Association Test: I. An improved scoring algorithm. Journal of Personality and Social Psychology, 85, 197-216. 
Greenwald, A. G., Poehlman, T. A., L., U. E., \& Banaji, M. R. (2009). Understanding and using the Implicit Association Test: III. Meta-analysis of predictive validity. Personality and Social Psychology Bulletin, 97, 14-41.

Griffiths, L. J., Parsons, T. J., \& Hill, A. J. (2010). Self-esteem and quality of life in obese children and adolescents: A systematic review. International Journal of Pediatric Obesity, 5, 282-304.

Grilo, C. M., White, M. A., \& Masheb, R. M. (2012). Significance of overvaluation of shape and weight in an ethnically diverse sample of obese patients with binge-eating disorder in primary care settings. Behaviour Research and Therapy, 50, 298-303.

Gumble, A., \& Carels, R. (2012). The harmful and beneficial impacts of weight bias on wellbeing: The moderating influence of weight status. Body Image, 9, 101-107.

Hilbert, A., Pike, K. M., Wilfley, D. E., Fairburn, C. G., Dohm, F. A., \& Striegel-Moore, R. H. (2011). Clarifying boundaries of binge eating disorder and psychiatric comorbidity: A latent structure analysis. Behaviour Research and Therapy, 49, 202211.

Hilbert, A., \& Tuschen-Caffier, B. (2010). Essanfälle und Adipostas: Ein Manual zur kognitiv-behavioralen Therapie der Binge-Eating-Störung [Binge Eating and Obesity: Cognitive-Behavioral Therapy Manual for Binge Eating Disorder]. Goettingen, Germany: Hogrefe.

Hilbert, A., Tuschen-Caffier, B., Karwautz, A., Niederhofer, H., \& Munsch, S. (2007). Eating Disorder Examination-Questionnaire: Evaluation der deutschsprachigen Übersetzung [Eating Disorder Examination-Questionnaire: Evaluation of the German version.]. Diagnostica, 53, 144-154. 
Hilbert, A., de Zwaan, M., \& Brähler, E. (2012). How frequent are eating disturbances in the population? Norms of the Eating Disorder Examination-Questionnaire, PLOS ONE, 7(1), e29125.

Hilbert, A., Tuschen-Caffier, B., \& Ohms, M. (2004). Eating Disorder Examination: Deutschsprachige Version des strukturierten Essstörungsinterviews [Eating Disorder Examination-Questionnaire: Evaluation of the German version.]. Diagnostica, 50, 98-106.

Hoffmeister, K., Teige-Mocigemba, S., Blechert, J., Klauer, K. C., \& Tuschen-Caffier, B. (2010). Is implicit self-esteem linked to shape and weight concerns in restrained and unrestrained eaters? Journal of Behavior Therapy and Experimental Psychiatry, 41, $31-38$.

Hofmann, W., Gawronski, B., Gschwendner, T., Le, H., \& Schmitt, M. (2005). A metaanalysis on the correlation between the implicit association test and explicit selfreport measures. Personality and Social Psychology Bulletin, 31, 1369-1385.

Karpinski, A. (2004). Measuring self-esteem using the Implicit Association Test: The role of the other. Personality and Social Psychology Bulletin, 30, 22-34.

Myers, A., \& Rosen, J. C. (1999). Obesity stigmatization and coping: Relation to mental health symptoms, body image, and self-esteem. International Journal of Obesity, 23, 221-230.

Risch, A. K., Buba, A., Birk, U., Morina, N., Steffens, M. C., \& Stangier, U. (2010). Implicit self-esteem in recurrently depressed patients. Journal of Behavior Therapy and Experimental Psychiatry, 41, 199-206.

Rosenberg, N., Bloch, M., Avi, I. B., Rouach, V., Schreiber, S., Stern, N., \& Greenman, Y. (2013). Cortisol response and desire to binge following psychological stress: Comparison between obese subjects with and without binge eating disorder. Psychiatry Research, 208(2), 156-161. 
Rudolph, A., Schröder-Abé, M., Schütz, A., Gregg, A. P., \& Sedikides, C. (2008). Through a Glass, Less Darkly? European Journal of Psychological Assessment, 24, 273-281.

Rudolph, A., Schröder-Abé, M., Riketta, M., \& Schütz, A. (2010). Easier when done than said: Implicit self-esteem predicts observed or spontaneous behavior, but not selfreported or controlled behavior. Journal of Psychology, 218, 12-19.

Schröder-Abé, M., Rudolph, A., \& Schütz, A. (2007). High implicit self-esteem is not necessarily advantageous: Discrepancies between explicit and implicit self-esteem and their relationship with anger expression and psychological health. European Journal of Personality, 21, 319-339.

Strack, F., \& Deutsch, R. (2004). Reflective and impulsive determinants of social behavior. Personality and Social Psychology Review, 8, 220-247.

Vartanian, L. R., Polivy, J., \& Herman, C. P. (2004). Implicit cognitions and eating disorders: Their application in research and treatment. Cognitive and Behavioral Practice, 11, 160-167.

Vater, A., Schröder-Abé, M., Schutz, A., Lammers, C. H., \& Roepke, S. (2010). Discrepancies between explicit and implicit self-esteem are linked to symptom severity in borderline personality disorder. Journal of Behavior Therapy and Experimental Psychiatry, 41, 357-364.

von Collani, G., \& Herzberg, P. Y. (2003). Eine revidierte Fassung der deutschsprachigen Skala zum Selbstwertgefühl von Rosenberg [A revised version of the German Rosenberg Self-Esteem Scale]. Zeitschrift für Differentielle und Diagnostische Psychologie, 24, 3-7.

Watts, K., \& Cranney, J. (2009). The nature and implications of implicit weight bias. Current Psychiatry Reviews, 5, 110-126. 
Wiers, R. W., Teachman, B. A., \& De Houwer, J. (2007). Implicit cognitive processes in psychopathology: An introduction. Journal of Behavior Therapy and Experimental Psychiatry, 38, 95-104.

Wilfley, D. E., Pike, K. M., Striegel-Moore, R. H. (1997). Toward an integrated model of risk for binge eating disorder. Journal of Gender, Culture, and Health, 2, 1-32.

Wilson, T. D., Lindsey, S., \& Schooler, T. Y. (2000). A model of dual attitudes. Psychological Review, 107, 101-126.

Wonderlich, S. A., Gordon, K. H., Mitchell, J. E., Crosby, R. D., \& Engel, S. G. (2009). The validity and clinical utility of binge eating disorder. International Journal of Eating Disorders, 42, 687-705. 
IMPLICIT COGNITIVE PROCESSES

\section{TABLES}

Table 1. Sample characteristics and group differences in psychopathology, explicit, and implicit measures in all groups.

\begin{tabular}{|c|c|c|c|c|c|c|}
\hline & BED & OB & $\mathrm{CG}$ & & & \\
\hline & $M(S D)$ & $M(S D)$ & $M(S D)$ & $F$ & Sig. $(p)$ & $\operatorname{ES}\left(\eta^{2}\right)$ \\
\hline Age (years) & 34.77 (10.29) & $35.19(11.08)$ & $34.65(10.70)$ & 0.67 & 0.48 & 0.03 \\
\hline Education (years) & $11.19(1.06)$ & $11.31(1.09)$ & $11.65(0.85)$ & 2.78 & 0.08 & 0.10 \\
\hline BMI $\left(\mathrm{kg} / \mathrm{m}^{2}\right)$ & $40.19(6.18)^{\mathrm{a}}$ & $40.27(6.08)^{\mathrm{a}}$ & $22.59(1.73)^{\mathrm{b}}$ & 188.83 & $<0.001$ & 0.88 \\
\hline \multicolumn{7}{|l|}{ EDE-Q } \\
\hline Restraint & $1.92(1.23)^{\mathrm{a}}$ & $1.48(1.47)^{\mathrm{a}, \mathrm{c}}$ & $1.04(1.30)^{\mathrm{b}, \mathrm{c}}$ & 3.08 & 0.06 & 0.11 \\
\hline Eating concern & $2.75(1.14)^{\mathrm{a}}$ & $0.84(0.76)^{\mathrm{b}}$ & $0.25(0.45)^{\mathrm{c}}$ & 59.49 & $<0.001$ & 0.70 \\
\hline Weight concern & $3.45(1.19)^{\mathrm{a}}$ & $2.32(1.15)^{\mathrm{b}}$ & $0.95(1.25)^{\mathrm{c}}$ & 32.69 & $<0.001$ & 0.57 \\
\hline Shape concern & $3.93(1.18)^{\mathrm{a}}$ & $2.74(1.30)^{\mathrm{b}}$ & $1.14(1.29)^{\mathrm{c}}$ & 42.35 & $<0.001$ & 0.63 \\
\hline Global score & $3.01(1.05)^{\mathrm{a}}$ & $1.84(1.01)^{b}$ & $0.84(0.96)^{\mathrm{c}}$ & 35.44 & $<0.001$ & 0.59 \\
\hline BSI & $67.23(11.68)^{\mathrm{a}}$ & $54.96(8.24)^{\mathrm{b}}$ & $52.38(11.29)^{\mathrm{b}}$ & 18.73 & $<0.001$ & 0.43 \\
\hline RSES & $27.00(7.08)^{\mathrm{a}}$ & $32.54(45.07)^{\mathrm{b}}$ & $34.31(5.24)^{\mathrm{b}}$ & 14.81 & $<0.001$ & 0.37 \\
\hline SSI & $1.56(0.69)^{\mathrm{a}}$ & $1.16(0.78)^{\mathrm{b}}$ & $0.27(0.31)^{\mathrm{c}}$ & 48.29 & $<0.001$ & 0.66 \\
\hline SE-IAT & $0.29(0.40)^{\mathrm{a}}$ & $0.50(0.26)^{\mathrm{a}, \mathrm{c}}$ & $0.52(0.27)^{\mathrm{b}, \mathrm{c}}$ & 4.05 & 0.03 & 0.14 \\
\hline WB-IAT & $0.22(0.55)^{\mathrm{a}}$ & $0.02(0.50)^{\mathrm{a}, \mathrm{b}}$ & $0.45(0.48)^{\mathrm{a}, \mathrm{c}}$ & 4.09 & 0.03 & 0.14 \\
\hline
\end{tabular}

Note. $N=26$ per group. Sig. $=$ significance; $p=$ probability; $F=$ F-statistic; $\mathrm{ES}=$ effect size;

$\mathrm{BMI}=$ body mass index in $\mathrm{kg} / \mathrm{m}^{2} ; \mathrm{EDE}-\mathrm{Q}=$ Eating Disorder Examination-Questionnaire; BSI

$=$ Brief Symptom Inventory; RSES = Rosenberg Self-Esteem Scale; SSI = Stigmatizing

Situations Inventory; SE-IAT $=$ Self-Esteem Implicit Association Test; WB-IAT $=$ Weight

Bias Implicit Association Test. ${ }^{\text {a,b,c }}$ Different superscripts indicate significant group differences. 
IMPLICIT COGNITIVE PROCESSES

Table 2. Prediction of global eating disorder psychopathology (EDE-Q) by dummy coded group membership, implicit and explicit self-esteem, implicit and explicit weight bias.

\begin{tabular}{lccccc}
\hline & $B$ & $S E$ & $\beta$ & $T$ & Sig. $(p)$ \\
\hline Step 1 & & & & \\
Constant & 0.84 & 0.20 & & 4.27 & $<0.001$ \\
Dummy CG-BED & 2.17 & 0.28 & 0.77 & 7.76 & $<0.001$ \\
Dummy CG-OB & 1.00 & 0.28 & 0.36 & 3.58 & 0.001 \\
Step 2 & & & & & \\
Constant & 2.49 & 0.67 & & 3.72 & $<0.001$ \\
Dummy CG-BED & 1.92 & 0.30 & 0.68 & 6.49 & $<0.001$ \\
Dummy CG-OB & 0.92 & 0.26 & 0.33 & 3.59 & 0.001 \\
SE-IAT & 0.86 & 0.34 & 0.21 & 2.57 & 0.01 \\
RSES & -0.06 & 0.02 & -0.30 & -3.40 & 0.001
\end{tabular}

Step 3

$\begin{array}{lccccc}\text { Constant } & 2.37 & 0.72 & & 3.31 & 0.001 \\ \text { Dummy CG-BED } & 1.83 & 0.36 & 0.65 & 5.12 & <0.001 \\ \text { Dummy CG-OB } & 0.86 & 0.32 & 0.31 & 2.74 & 0.01 \\ \text { SE-IAT } & 0.87 & 0.34 & 0.21 & 2.53 & 0.01 \\ \text { RSES } & -0.06 & 0.02 & -0.29 & -3.10 & 0.003 \\ \text { WB-IAT } & 0.07 & 0.21 & 0.03 & 0.32 & 0.75 \\ \text { SSI } & 0.10 & 0.18 & 0.06 & 0.51 & 0.59\end{array}$

Note. $N=78 . B=$ unstandardized coefficients; $S E=$ standard error; $\beta=$ standardized coefficient; $T=$ t-statistic; Sig. $=$ significance; $p=$ probability; SE-IAT $=$ Self-Esteem Implicit Association Test; RSES = Rosenberg Self-Esteem Scale; WB-IAT = Weight-Bias Implicit Association Test; SSI = Stigmatizing Situations Inventory. 
IMPLICIT COGNITIVE PROCESSES

ACKNOWLEDGMENTS, DECLARATION OF INTEREST, AND ROLE OF FUNDING

\section{ORGANIZATIONS}

This work was supported by the Federal Ministry of Education and Research (BMBF), Germany, FKZ: 01GV0601 and 01EO1001. We are grateful to Elizabeth Anne Bollinger, M.Sc. for her providing of language help and proof reading the manuscript. No author of this manuscript does have any conflict of interest, financial or otherwise, related to the submitted work. 